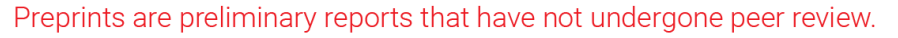 \\ They should not be considered conclusive, used to inform clinical practice, or referenced by the media as validated information. \\ Fabrication Large-scale Diffractive Lens Arrays on Chalcogenide Glass by Means of Step-and-Repeat Hot Imprinting and Non-Isothermal Glass Molding
}

\section{Yang Shu}

Hunan University

\section{Tiantong Chen}

The Ohio State University

Wenchen Zhou

The Ohio State University

zhixiong Zhou

Hunan University

Allen Y Yi ( $\nabla$ yi.71@osu.edu )

The Ohio State University

\section{Research Article}

Keywords: Chalcogenide glass, diffractive lens array, PDMS mold, hot imprinting, non-isothermal molding

Posted Date: February 12th, 2021

DOl: https://doi.org/10.21203/rs.3.rs-227206/v1

License: (c) (1) This work is licensed under a Creative Commons Attribution 4.0 International License. Read Full License

Version of Record: A version of this preprint was published at The International Journal of Advanced Manufacturing Technology on July 16th, 2021. See the published version at https://doi.org/10.1007/s00170-021-07716-w. 


\section{Abstract}

In this study, a new cost-effective and high-precision process chain for the fabrication of large-scale diffractive lens arrays on chalcogenide glass is proposed. First, a positive diffractive lens array is fabricated on a PMMA master substrate by employing a step-and-repeat hot imprinting process. The direct hot imprinting can transfer the microstructures from a heated mold to the polymer substrate accurately. Repeating the hot imprinting process according to a predetermined path, the desired diffractive lens array is obtained. Unlike photolithography and electron-beam writing, which are expensive technologies with sophisticated process, the hot imprinting is an easier, cheaper and more eco-friendly method for fabricating diffractive features with continuous profile. Afterwards a casting process is applied to create a PDMS mold with the negative features. The diffractive lens array with continuous profile is successfully transferred from the master substrate to the PDMS elastomer, which is used as a mold for subsequent precision glass molding. Finally, the microstructures of PDMS mold are replicated to the chalcogenide glass by non-isothermal glass molding. In this process, the mold and workpiece are set at different temperatures. The PDMS mold at low temperature maintains enough rigidity, so as to press the features into the softened chalcogenide glass more easily, which is at relatively higher temperature, resulting in a positive high-fidelity diffractive lens array on the chalcogenide glass. Surface profiles and optical performance of the fabricated components are characterized quantitatively. Results showed that large-scale diffractive lens array with continuous profile can be successfully fabricated on Chalcogenide glass by this proposed process chain with high quality and integrity.

\section{Introduction}

Diffractive lens arrays are important optical components in various applications, such as integral imaging, concentrator photovoltaics and LED lighting [1-4]. The diffractive lens arrays are usually composed of a same diffractive lens feature in multiform arrangements. The focusing and imaging performance of diffractive lens depends on the form accuracy and surface finish of its feature. Many methods for the manufacture of diffractive lens arrays have been extensively investigated, including laser direct writing [5-7], focused ion beam [8], and photolithography [9]. By increasing the number of orders, the optical properties of the multiorder diffractive optical elements fabricated by lithography can be obviously improved. However, due to the influence of the straight edge diffraction of the binary structure, the light cannot be concentrated in the far field. Thus, in theory, the focusing ability obtained by discretestep profile cannot be compared with that of the continuous profile. The continuous profile can be machined by laser direct writing and focused ion beam technology [6], but the pursuit of surface quality while maintaining a high machining efficiency in those processes has proven to be challenging. In general, most of these techniques require expensive equipment and involve complicated process steps, in addition, these processes are usually time consuming as well. Accordingly, they are not the perfect solutions for manufacturing large-area diffractive array optics with intricate shapes, particularly continuous profiles. 
Precision glass molding is a high-efficiency and low-cost manufacturing process, which can easily cope with the manufacture of optical elements in macroscopic and micro/nano scale [10-11]. It is becoming a preferred alternative method for high volume production of plastic and glass optics. In precision glass molding, the geometrical accuracy and surface quality of the workpieces are basically determined by the mold. Therefore, it is the critical step to fabricate the precision master mold with inverse microstructure. At present, the material removal methods, such as precision grinding, polishing and diamond turning still play a dominant role in mold fabrication. Hard materials, such as metals (e.g. Ni), SiC and WC, are usually the popular choices for precision mold, due to the excellent physical properties at high temperature and inert chemistry. However, higher the hardness a material has, more difficult the machining process is. Especially for the large area micro/nano-scale structure arrays, the uniformity of the whole array is difficult to maintain due to tool wear. In addition, fabrication of intricate microstructures with sharp angles on hard materials, such as the diffractive lens array with continuous profile in this research, is also a technical challenge using existing manufacturing processes [12-13]. In addition, initial cost for machining such features should also be considered. These issues limit the wide applications of precision compression molding in replicating high resolution and complex surfaces.

These limitations of the hard material mold in machining, in principle, can be overcome by using soft material molds. Recently, polydimethylsiloxane (PDMS), one of the soft material molds, has been widely adopted as the mold for precision molding, due to its simple, mature and low-cost manufacturing process, as well as the flexible mechanical properties [14-15]. Compared with the hard material, PDMS has the following advantages as a mold: (1) PDMS can be easily obtained over large areas with good reproducibility and high accuracy, by casting a liquid onto a master substrate followed by thermal curing. (2) PDMS has low surface energy, which is convenient to separate from workpiece, and does not damage the surfaces of workpiece during separation stage. (3) PDMS mold is less sensitive to surface defects and contamination. However, the PDMS, as a precision mold, also encounters some problems. The first one is the difficulty of fabricating master substrate with large-area intricate features. Inkjet printing technologies is a popular flexible fabrication method of microlens array, but the structures of the microlens are limited to spherical and aspheric shapes [16]. Other sophisticated technologies, including photolithography, e-beam lithography and wet-etching process [17-18], are good at creating complex features with high precision. But their process is extremely complex and costly. The second one is the poor rigidity of PDMS. The rigidity of PDMS is lower than that of chalcogenide glass at room temperature, and decrease with the increase of working temperature [19]. Although the rigidity can be improved by changing the components of pre-curing PDMS [20] and adjusting the manufacturing parameters of PDMS, such as mixing ratio [21], curing time, and thermal ageing temperature [22-24], but these enhanced methods have limited performance improvements. In addition, by increasing the molding temperature [25], the workpiece can be softened to the point that it can slip into the mold cavities without much pressure. As expected, the deformation of the PDMS mold was almost avoided in the molding stage under this condition. However, high temperature leads to the loss of sulfur elements in the chalcogenide glass [26], which directly affects the optical properties of the lens. And the higher the working temperature, the shorter the life of mold. Therefore, although the existing manufacturing 
methods can replicate the features of PDMS mold to chalcogenide glass, there is an urgent demand for introducing a new manufacturing process with better energy efficient, stable and longer life of the mold.

Focusing on the above problems, in this paper, a fast and low-cost process chain of inscribing large-area diffractive lens arrays on chalcogenide glass by combining step-and-repeat hot imprinting and nonisothermal compression molding is proposed and investigated. Three major process steps are needed: the first step is the fabrication of a diffractive lens array on plastic master substrate employing hot imprinting. A single diffractive feature with continuous profile is machined on a brass mold by diamond turning. The continuous profile diffractive feature is copied completely to the polymer substrate by hot imprinting consecutively until the entire array is obtained. The direct hot imprinting ensures that the continuous features of the mold is replicated with high fidelity, and the accuracy of the entire array can be guaranteed by the stability of imprinting temperature and the high motion accuracy of the servo platform. At the end of this process a precise PDMS mold is generated by pouring a well-prepared PDMS liquid into the master substrate followed with curing. Lastly, the diffractive lens array is fully replicated on chalcogenide glass using non-isothermal glass molding. The non-isothermal molding process allows the PDMS mold to remain rigidity at lower temperature, to press into the softened chalcogenide glass at relatively high temperature. To demonstrate the feasibility of the proposed process chain, a $30 \times 30$ square diffractive lens array was fabricated on $\mathrm{As}_{2} \mathrm{~S}_{3}$ glass. Each fabrication step and the resulting accuracy are carefully described throughout the whole process chain. The results showed that the proposed method proved has potentials for manufacturing optics elements with better optical performance and a much lower cost and higher efficiency.

\section{Material And Method}

In this section, we present the material and method of the whole process chain. The material of master substrate is selected as PMMA, which has a low transition temperature, suitable for hot imprinting in large area. Chalcogenide glass used in the experiment is AMITIR-6 $\left(\mathrm{As}_{2} \mathrm{~S}_{3}\right)$, and the PDMS prepolymer is prepared from an elastomer kit (Sylgard 184, Dow Corning). The thermal and mechanical properties of PMMA, PDMS and $\mathrm{As}_{2} \mathrm{~S}_{3}$ are shown in Table 1. 
Table 1

Material properties and parameters

\begin{tabular}{|c|c|c|c|}
\hline Material Properties & PMMA & PDMS & $\mathrm{As}_{2} \mathrm{~S}_{3}$ \\
\hline Density, $\rho /\left(\mathrm{kg} \cdot \mathrm{m}^{-3}\right)$ & 1,190 & 965 & 3,200 \\
\hline Elastic modulus, E/Pa & $3.3 E+9$ & $1.50 E+6$ & $1.58 \mathrm{E}+10$ \\
\hline Poisson's ration, $v$ & 0.3 & 0.5 & 0.24 \\
\hline Thermal conductivity, $k_{\mathrm{c}} /\left[\mathrm{W} \cdot(\mathrm{m} \cdot \mathrm{k})^{-1}\right]$ & 0.19 & 0.15 & 0.167 \\
\hline Coefficient of Thermal Expansion, $a$ & 6.0E-05 & $9.6 \mathrm{E}-04$ & $2.14 \mathrm{E}-05$ \\
\hline Specific Heat, $C_{\mathrm{p}}\left(\mathrm{J} / \mathrm{kg}^{\circ} \mathrm{C}\right)$ & 1,466 & 1,460 & 456.36 \\
\hline Transition Temperature, $T_{\mathrm{g}} /{ }^{\circ} \mathrm{C}$ & 105 & & 180 \\
\hline Softening Point, $T_{\mathrm{g}} /{ }^{\circ} \mathrm{C}$ & & & 208 \\
\hline \multicolumn{4}{|c|}{$\begin{array}{l}\text { The whole process chain is illustrated in Fig. 1, as following, (a) Step-and-repeat hot imprinting is } \\
\text { introduced to duplicate a single diffractive feature repeatedly on substrate, creating a diffractive lens } \\
\text { array on PMMA master substrate. (b) The diffractive lens array on PMMA master substrate is then } \\
\text { transferred successfully onto the PDMS mold by using a casting process. (c) Non-isothermal } \\
\text { precision glass molding is used to replicate the entire features form the PDMS mold to chalcogenide } \\
\text { glass. }\end{array}$} \\
\hline
\end{tabular}

\subsection{Step-and-repeat hot imprinting}

The PMMA master substrate was fabricated by using step-and-repeat hot imprinting. In this process, a single diffractive feature with continuous profile was firstly machined on a brass mold by single point diamond turning. The continuous profile diffractive feature was then copied completely to the polymer substrate by imprinting consecutively until an entire array is obtained. As shown in Fig. 2, the imprinting process of the diffractive lens array was conducted on an ultraprecision 3-axis CNC turning machine. A self-developed imprinting device was mounted on the tool post, which has a sub-micron scale motion accuracy in $z$ axis. The polymer substrate was clamped on the center of a vacuum chuck, which follows the servo motions along both $\mathrm{x}$ and $\mathrm{y}$ axes. In addition, equipped with a band heater and a $K$ type temperature sensor, the imprinting device can achieve rapid heating and accurate temperature control during hot imprinting process.

The manufacturing procedure works for diffractive lens array as follows: first, the brass mold temperature is heated to $130{ }^{\circ} \mathrm{C}$ by the band heater and maintained for 10 minutes, while the PMMA substrate is kept at room temperature. The heated mold is then imprinted onto the PMMA substrate with $2 \mu \mathrm{m}$ depth of pressing and maintained for 2 seconds. Since $z$ axis of the turning machine was equipped with 
nanometer motion precision, $2 \mu \mathrm{m}$ imprinting depth can ensure the microlens cavities are completely filled. After one diffractive lenslet was fabricated, the PMMA substrate moves to the next position for the next lenslet fabrication. By repeating the above process, a desired pattern lens array is finally obtained on the PMMA substrate. Relevant hot imprinting parameters are illustrated in Table 2. These parameters have been optimized based on previous hot imprinting experiments of the same material. The direct hot imprinting method ensures that the continuous features of the mold can be replicated with high fidelity, and the accuracy of the entire array is guaranteed by the hot imprinting process and the high precision motion system. Furthermore, the hot imprinting process is carried out at room temperature, and the polymer substrate does not need to be heated and cooled repeatedly, which greatly reduces the thermal cycle time and energy consumption, demonstrating that the proposed process for fabrication of continuous profile diffractive lens arrays on polymer substrate is robust and cost-effective method.

Table 2

Parameters for hot imprinting

\begin{tabular}{|ll|}
\hline Imprinting Parameters & Values \\
\hline Temperature of the mold, $T_{\mathrm{m}}$ & $130{ }^{\circ} \mathrm{C}$ \\
\hline Imprinting rate, $C_{\mathrm{i}}$ & $0.5 \mu \mathrm{m} \mathrm{s}^{-1}$ \\
\hline Imprinting depth, $D_{\mathrm{i}}$ & $2 \mu \mathrm{m}$ \\
\hline Holding time, $T_{\mathrm{h}}$ & $5 \mathrm{~s}$ \\
\hline
\end{tabular}

\subsection{PDMS casting}

PDMS has been widely adopted as the mold as mature, versatile and low-cost manufacture process. PDMS also has stable mechanical properties. To create a flexible mold for precision glass molding, a casting process is employed to generate the PDMS mold, and the imprinted PMMA master is used as the casting substrate. PDMS prepolymer was prepared from an elastomer kit (Sylgard 184, Dow Corning), which consists of a pre-polymer base (part A) and a crosslinking curing agent (part B). Relevant process parameters are illustrated in Table 3. Detailed manufacture process is as follows. First, mix two parts in a beaker thoroughly for 5 minutes. The ratio of the two parts is $5: 1$ as recommended for high rigidity and hardness [21]. Fully mixed liquid PDMS was poured directly into the PMMA master substrate to form a negative replica of the microstructures. A circular frame was attached to the PMMA substrate serving as a boundary to shape the PDMS mold into a disk shape and also prevent leakage during curing. Then, the formed mold was thoroughly degassed in low vacuum for 20 minutes until all bubbles were removed. The PDMS mold is cured using a precision heating furnace with temperature control. Since the mechanical properties of PDMS are closely related to the curing temperature and time, to get better rigidity and hardness, the operating temperature of $150{ }^{\circ} \mathrm{C}$ and a curing time of 10 minutes are chosen as the curing parameters [22]. Finally, a cured PDMS mold, patterned with diffractive lens array features on one side, was separated from the PMMA master substrate. 
Table 3

Parameters for PDMS casting process

\section{Material PDMS \\ Ratio $\quad 5: 1$ \\ Stirring speed $\quad 100 \mathrm{rpm}$ \\ Cure temperature, $T_{C} \quad 150{ }^{\circ} \mathrm{C}$ \\ Cure time, $T_{\mathrm{s}} \quad 10 \mathrm{~min}$ \\ Degassed time $20 \mathrm{~min}$ \\ 2.3 Non-isothermal precision glass molding}

With the PDMS mold completed, the next step is to copy the microstructures on the PDMS mold to the chalcogenide glass. The Young's modulus of PDMS is around 2.59 MPA at room temperature [19], which is lower than the conventional polymer. The value will significantly decrease with the increase of working temperature. Therefore, it is a challenge to ensure that the features of PDMS mold are completely pressed into the glass substrate. In view of this, a non-isothermal molding process is proposed in this section. The workpiece and the mold are set at different temperatures. The PDMS mold is kept at a low temperature to maintain a better stiffness and thermal expansion rate, while the workpiece is heated to a higher temperature, above its transition temperature for molding. Stiffer molds will have less deformation and improve replication fidelity. The mold at lower temperature is then pressed on the glass substrate that was kept at high temperature, completing the transfer of the surface features. Due to the low thermal conductivity of PMDS and glass material, as well as the short molding time for micro-scale structures, temperature gradient in the contact interface between glass and mold during molding stage remains low. As expected, the small temperature difference between mold and glass does not have much effect on the rigidity of PDMS mold and the viscosity of the glass. The microstructures of PDMS mold can be replicated to the glass substrate with high integrity as demonstrated next.

The feasibility of this method is tested by molding a $30 \times 30$ square diffractive lens array from PDMS mold to chalcogenide glass. The experiment was conducted on a precision glass molding machine (OSUPGM100), as shown in Fig. 3. The molding device consists of a rapid heating unit, a loading unit, and is liquid-cooled. The unit can also be operated under vacuum or with nitrogen. The heating in this press is divided into upper and lower heating components, and the molds are mounted in the middle of the upper and lower heaters. The lower heater is fixed while the upper heater can move vertically with the pressure loading system to work with different thickness of the workpieces. Based on heat conduction, heating is provided by ten cartridge heaters with a total power of 5,000 W. Equipped with precise thermocouple and temperature controller, the rapid heating system can achieve real time closed-loop control of the temperature in a wide range from $26^{\circ} \mathrm{C}$ to $900^{\circ} \mathrm{C}$, with the accuracy of $0.1^{\circ} \mathrm{C}$. An integrated linear 
actuator provides loading pressure for smooth vertical movement of the upper heater with a micron-level precision. The maximum load is $3,800 \mathrm{~N}$. In addition, the pressure loading system combines the bidirectional force sensor and the linear optical encoder to realize duel closed-loop control of position and pressure. The molding device constructed with the above configuration is capable to mold optical elements of various shapes and materials.

Table 4

Parameters for non-isothermal molding process.

\begin{tabular}{|ll|}
\hline Imprinting Material & $\mathrm{As}_{2} \mathrm{~S}_{\mathbf{3}}$ \\
\hline Temperature of the PDMS mold & $150{ }^{\circ} \mathrm{C}$ \\
\hline Temperature of the $\mathrm{As}_{2} \mathrm{~S}_{3}$ substrate & $200{ }^{\circ} \mathrm{C}$ \\
\hline Heating rate & $3{ }^{\circ} \mathrm{C} \mathrm{s}^{-1}$ \\
\hline Imprinting rate & $0.5 \mu \mathrm{m} \mathrm{s}^{-1}$ \\
\hline Imprinting depth & $1.2 \mu \mathrm{m}$ \\
\hline
\end{tabular}

The implementation steps of non-isothermal glass molding are as follows: first, the PDMS mold is fixed in the center of the upper heating plate, and the $\mathrm{As}_{2} \mathrm{~S}_{3}$ glass is installed in the lower heating plate directly below the mold. A vacuum pump is used to purge the working area, and the temperatures of the upper and lower heating plate are heated to $150{ }^{\circ} \mathrm{C}$ and $200{ }^{\circ} \mathrm{C}$ respectively. Second, after the PDMS mold and the $\mathrm{As}_{2} \mathrm{~S}_{3}$ glass are heated to the designated temperatures, the mold is slowly pressed into the glass sample at a speed of $0.5 \mu \mathrm{m} / \mathrm{s}$ and a displacement of $1.2 \mu \mathrm{m}$ under the precise displacement control. The molding pressure was $200 \mathrm{~N}$. Finally, when the pressure was reduced to a stable value, the mold was moved upward and slowly separated from the glass. Accordingly, the microstructure on the PDMS mold is replicated to the $\mathrm{As}_{2} \mathrm{~S}_{3}$ glass. Relevant non-isothermal molding parameters are illustrated in Table 4.

\section{Results And Discussion}

\subsection{Characterization of the PMMA master substrate and PDMS mold}

For characterizing the microstructures of diffractive lens array on the PDMS mold, the imprinted PMMA master substrate and the PDMS mold are evaluated. The photographs of imprinted PMMA master substrate and the PDMS mold are shown in Fig. 4 (a) and 4 (c), respectively. The central regions of the patterns are captured by optical microscope and shown in Fig. 4 (b) and 4 (d). In the photographs, a $30 \times 30$ diffractive lens array is closely aligned in the form of a square. The outer edge of each lenslet is adjacent immediately to that of the others, showing a high spatial filling rate. The two arrays are clearly formed, no visible scratches, damage, deformation, and incomplete features were discovered on the 
surface. It indicates that a uniform diffractive lens array covering the relatively large area is formed on PMMA and PDMS surface.

To evaluate the uniformity of the diffractive lens array, an arbitrary region of $2 \times 2$ lenslets is further captured by white-light interferometer (Wyko NT9100, Bruckner, Tucson, Arizona, USA). Figure 5 (a) shows the 3D structure of the PMMA master substrate. The diffractive lenslets, which have the same microstructures, are uniformly distributed on the view. There is no obvious deformation or defects on the array. Although a portion of the material in the outermost area of the lenslet is raised due to the extrusion of the mold during imprinting. However, the convex area is very small, accounting for a small proportion of the lens, so the effect on the optical performance is almost negligible. Figure 5 (c) presents the corresponding profile along the A-A direction (in Fig. 5(a)). The height of the lenslet is $1.2 \mu \mathrm{m}$ with $1 \mathrm{~mm}$ diameter, and the distance between two successive lenslets is $1 \mathrm{~mm}$. The diffractive lens features, including central circle and nine concentric grooves, are continuous, complete and symmetrical, showing the well-defined diffractive feature with continuous profile can be obtained by rapid imprinting process. Figure 5 (b) and (d) show the structures of the cured PDMS diffractive lens array and the corresponding cross-section profile, which exhibits the same features as the PMMA master substrate but with an inverse shape. As expected, the height of the lens and the distance between the lenses were measured, which were $1.2 \mu \mathrm{m}$ and $1 \mathrm{~mm}$, respectively, which were consistent with the PMMA master substrate. The features on PDMS are also continuous and complete, showing a good uniformity of the measured array. It confirmed that PDMS casting process replicates the characteristics on PMMA master substrate with high fidelity.

To investigate form accuracy of the lenslet, one randomly selected lenslet from the microlens array is captured and the cross-sectional profiles are measured and compared with each other. The lenslets on PMMA and PDMS are shown in Fig. 5 (e) and (f), respectively. In Fig. 5 (e) and (f), the contour on lenslet is very smooth and continuous, without breaks or steps, showing the advantages of the proposed process over the existing process. Furthermore, the cross-hair profiles have a good agreement with each other, showing no distortion of the lenslet. The height of the central curvature and other outer prism edges are $1.20 \mu \mathrm{m}$, matching the design geometry well. And the resulted geometrical errors are less than $0.1 \mu \mathrm{m}$.

\subsection{Characterization of the chalcogenide glass}

The feasibility of the proposal new process chain was demonstrated by generating a diffractive lens array with continuous profile on a chalcogenide glass. Similar to the aforementioned profile measurement, the microstructures on chalcogenide glass are characterized in detail, and the central regions of the patterns are captured by optical interferometer profiler. Figure 6 (a) and 6 (b) show the photograph and optical microscope with a magnification of $5 \times$ of the chalcogenide glass. The structures on the array are complete and consistent, and the arrangement of lenslet is completely consistent with the PDMS mold, showing a good uniformity. Furthermore, an arbitrary region of $2 \times 2$ diffractive lens array is further extracted, the 3D structure and the corresponding profile along the A-A direction are illustrated in Fig. 7 (c) and 7 (d), respectively. The lenslet features consist of a central circle and nine concentric rings, showing a perfectly continuous topography. The height of some grooves is a little lower than $1.2 \mu \mathrm{m}$, 
resulting in a certain shape error. The main reason for the error is that the thermal stress caused by the non-isothermal temperature distribution makes the lenslet shrink slightly. The maximum deviation of the height is $0.1 \mu \mathrm{m}$, which is deemed safe for optical performance.

In order to further evaluate the form accuracy of the lenslet, an arbitrary lenslet is extracted from Fig. 6 (c). The 3D structure of the lenslet and the cross-hair profiles are illustrated in Fig. 6 (e) and 6 (f), respectively. The two profiles match well with each other, showing no distortion of the lenslet. The feature height in the middle of lenslet is about $1.15 \mu \mathrm{m}$, slightly lower than that of the PDMS mold. It indicates that the flexible mold still has a little deformation under a certain mold pressure, although the adoption of non-isothermal molding process has improved the overall rigidity of the PDMS mold. In general, the continuous morphology and complete groove features of the array indicate that the soft PDMS mold features can be completely copied to the chalcogenide glass by non-isothermal compression molding.

\subsection{Optical setup and measurement}

To evaluate the shape uniformity and the focusing function of the diffractive lens array on chalcogenide glass, a simple optical setup was built and employed. As shown in Fig. 7 (a), it consists of an infrared source (HawkEye Technologies, Infrared Source IR-35, Milford, Connecticut, USA), a pair of polarizers, the molded diffractive lens array and an infrared camera (Sofradir EC, Inc., Fairfield, New Jersey, USA). The light source, whose wavelength range is around 6-14 $\mu \mathrm{m}$ based on the input power, provides a collimated infrared light beam using a parabolic reflector. During the experiment, the input power was controlled so that the wavelength of generated light source is around $8 \mu \mathrm{m}$. Since the intensity of light is high, we used two polarizers to reduce the infrared beam intensity. The molded diffractive lens array was placed after two polarizers to focus the incident light on the IR camera image plane. The spot pattern of the diffractive lens array and the focal point light intensity distribution of randomly selected a row of microlenslets are shown in Fig. 8(a) and 8(b), respectively. In the picture, the focal spots are clear and evenly distributed. The lens array successfully focused the incident plane waves into many convergent spherical waves. But there is some background noise in the spot pattern. The main reason is that the microlens array works in long wave infrared band. And all objects in the testing environment, including the sensor itself, are sources of background noise. However, Fig. 8(b) shows that the focal spots still possess good intensity profiles despite the serious noise level, which validates the focusing ability of these Fresnel lenses with continuous surface.

\section{Conclusions}

This paper provides a new manufacturing method by combining step-and-repeat imprinting, PDMS casting and non-isothermal glass molding for fabrication of continuous profile diffractive lens array. A square 30x30 diffractive lens array on chalcogenide glass was successfully obtained. Each diffractive lens consists of nine concentric prism rings, all of which are maintained at a height of $1.2 \mu \mathrm{m}$. In addition, the lens has a nearly perfect continuous profile, which matches well with the design, and the surface 
roughness can reach to nanoscale level. Moreover, the step-and-repeat imprinting method offers an economical, environmentally friendly and high-quality manufacturing choice for creating complex feature array on polymer substrates. The non-isothermal molding makes it possible to transfer the micro features of the soft mold (PDMS) to hard substrate ( $\mathrm{As}_{2} \mathrm{~S}_{3}$ glass) with high fidelity. Finally, the optical properties of As2S3 glass were evaluated using an optical testing setup. Optical performance of the diffractive lens array demonstrates a promising process for optics fabrication.

For future work, the protrusion in the outermost area of lens has to be reduced by optimizing the process parameters to keep all of the diffractive features to design, so as to increase the optical performance.

\section{Declarations}

\section{Acknowledgement}

This material is partially based on work supported by National Science Foundation under Grant No. 1537212. Any opinions, findings, and conclusions or recommendations expressed in this material are those of the authors and do not necessarily reflect the views of the National Science Foundation. Yang Shu also gratefully acknowledges the financial support from the China Scholarship Council (CSC). This work is also partially supported by the II-VI Foundation's Block Gift Program.

Authors' contributions Yang Shu, Zhixiong Zhou and Allen Yi contributed to the conceptualization, writing and editing. Tiantong Chen, Yang Shu and Wenchen Zhou contributed to the molding experiments, data analysis.

Availability of data and materials, all data generated or analyzed during this study are included in this published article.

Compliance with ethical standards:

Conflict of interest the authors declare that they have no conflicts of interest.

Ethical approval not applicable.

Consent to participate, yes, consent to participate from all the authors.

Consent to publish, yes, consent to publish from all the authors.

\section{References}

1. Li KY et al (2019) Dual-view display technology and device based on multi-focus Fresnel lens array SID Symp. Digest of Tech. Papers 50 622-5

2. Mathias Hain et al 2005 3D Integral Imaging Using Diffractive Fresnel lens Arrays Opt. Express 13 315-26 
3. Huang R, Zhang XQ, Ng BP, Kumar SA, Liu K 2019 Roll-to-roll embossing of optical radial Fresnel lenses on polymer film for concentrator photovoltaics: a feasibility study Int. J. of Precis. Eng. and Manuf. Green Tech. 10 1007-18

4. Wang GZ, Wang LL, Li FL, Kong D (2012) Design of optical element combining Fresnel lens with microlens array for uniform light-emitting diode lighting. J Opt Soc Am A 29:1877-1884

5. Zhang F et al (2020) Fabrication of Chalcogenide Glass Based Hexagonal Gapless Microlens Arrays via Combining Femtosecond Laser Assist Chemical Etching and Precision Glass. Molding Processes Materials 13:3490

6. Yan LY, Yang D, Gong QH, Li Y (2020) Rapid Fabrication of continuous profile Fresnel microlens array by femtosecond laser focal field engineering Micromachines $11112-20$

7. Roeder $\mathrm{M}$ et al (2019) Fabrication of curved diffractive optical elements by means of laser direct writing, electroplating, and injection compression molding. J Manuf Process 47:402-409

8. Fu YQ, Ngoi BKA (2001) Investigation of Diffractive-refractive Microlens Array Fabricated by Focused Ion Beam. Opt Eng 40:511-516

9. Aristizabal SL et al (2013) Microlens array fabricated by a low-cost grayscale lithography maskless system. Opt Eng 52:125101-125108

10. Liu X, Zhou T, Zhang L et al (2018) Fabrication of spherical microlens array by combining lapping on silicon wafer and rapid surface molding. J Micromech Microeng 28:075008-075017

11. Zhou T, Liu X, Liang Z, Liu Y, Xie J, Wang X 2017 Recent advancements in optical microstructure fabrication through glass molding process Front. Mech. Eng. 12 46-65

12. Liu X, Mo R, Li K et al (2020) Manufacturing of 3D Microlens Array Mold on Bulk Metallic Glass by Self-Aligned Multi-Ball Hot Embossing. Int J of Precis Eng Manuf-Green Tech 10:1007

13. Zhang L, Yi YA (2020) Manufacturing of a microlens array mold by a two-step method combining micro indentation and precision polishing. Appl Opt 59(23):6945-6952

14. Xiong $H$, Wang $L$, Wang $Z$ (2019) Chalcogenide microlens arrays fabricated using hot embossing with soft PDMS stamps. J Non-crystalline Solids 521:119542

15. Liu Y, Zhang P, Deng Y et al (2014) Polymeric microlens array fabricated with PDMS mold-based hot embossing. J Micromech Microeng 24:095028

16. Fang F, Tao X, Chen X et al 2020 Microlens Fabrication by Replica Molding of Electro-Hydrodynamic Printing Liquid Mold Micromachines 11161

17. Ostrovsky N, Yehuda D, Tzadka Sivan et al (2019) Direct imprint of optical functionalities on freeform chalcogenide glasses. Adv Optical Mater 7:1900652

18. Bian $\mathrm{H}$ et al (2016) Direct fabrication of compound-eye microlens array on curved surfaces by a facile femtosecond laser enhanced wet etching process. Appl Phys Lett 109:221109

19. Johnston ID, McCluskey DK, Tan CK, L, Tracey MC (2014) Mechanical characterization of bulk Sylgard 184 for microfluidics and microengineering. J Micromech Microeng 24:035017-035024 
20. Zhang YF, Ren YJ, Guo HC, Bai SL (2019) Enhanced thermal properties of PDMS composites containing vertically aligned graphene tubes. Appl Therm Eng 150:840-848

21. Khanafer K, Duprey A, Schlicht M, Berguer R (2009) Effects of strain rate, mixing ratio, and stressstrain definition on the mechanical behavior of polydimethylsiloxane (PDMS) material as related to its biological applications. Biomed Microdevice 11:503

22. Liu M, Sun J, Chen Q (2009) Influences of heating temperature on mechanical properties of polydimethylsiloxane. Sensors Actuators A:Physical 151:42-45

23. Liu M, Chen QF (2007) Characterization study of bonded and unbonded polydimethylsiloxane aimed for bio-microelectromechanical systems-related applications. J Micro/Nanolith MEMS MOEMS 6:023008

24. Kim M, Moon B, U, Hidrovo CH (2013) Enhancement of the thermo-mechanical properties of PDMS molds for the hot embossing of PMMA microfluidic devices. J Micromech Microeng 24:095024

25. Orava J, Kohoutek T, Greer A, Fudouzi H 2011 Soft imprint lithography of a bulk chalcogenide glass Opt. Mater. Express 1 796-802

26. Hu J, Feng N, Carlie N et al (2010) Optical loss reduction in high-index-contrast chalcogenide glass waveguides via thermal reflow. Opt Express 18:1469-1478

\section{Figures}

(a) Step-and-repeated hot imprinting

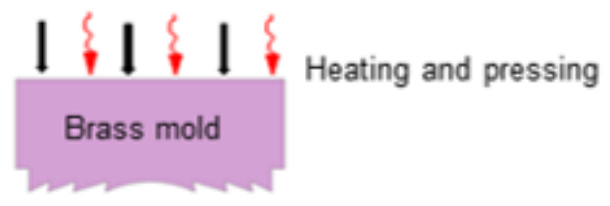

PMMA substrate

PMMA master substrate

(b) PDMS casting

Pouring and curing
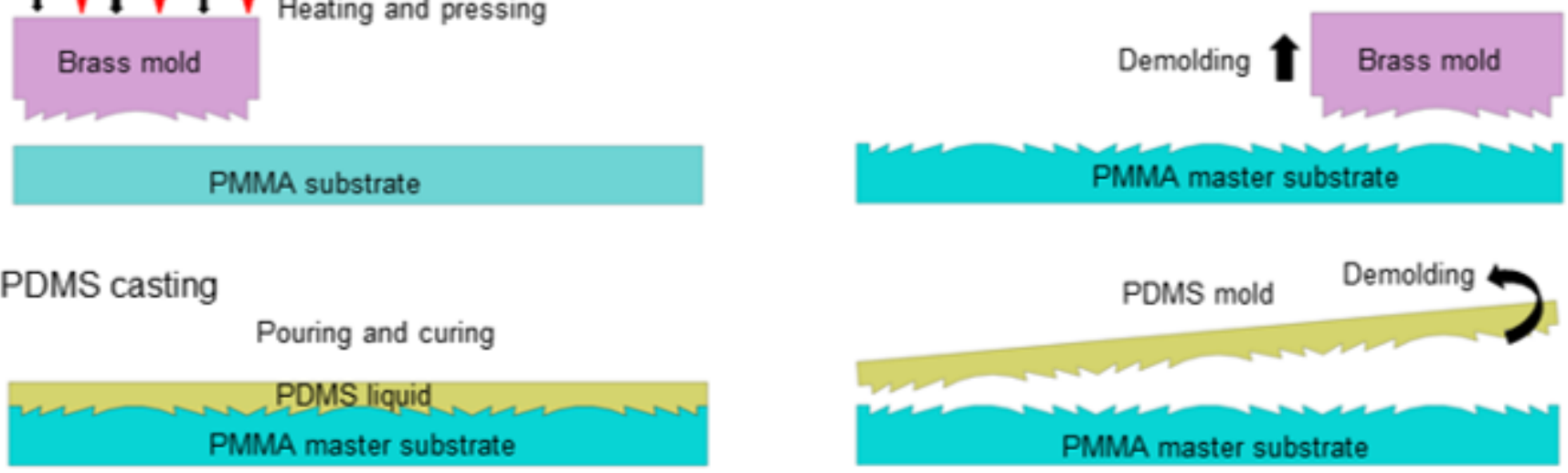

(c) Non-isothermal glass molding

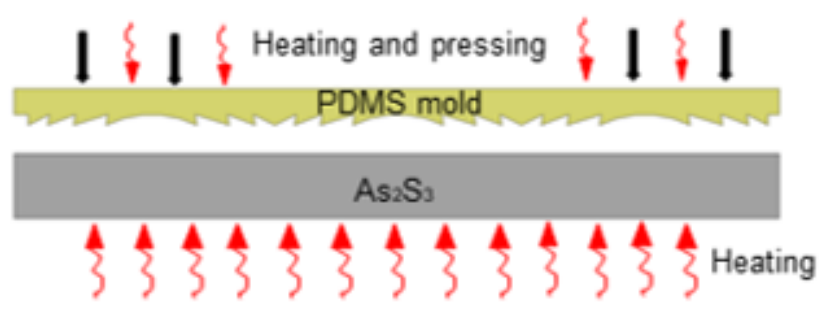

$\mathrm{As}_{2} \mathrm{~S}_{3} \mathrm{DOE}$ array

Figure 1 
Schematic of proposed fabrication process, (a) step-and-repeat hot imprinting, (b) PDMS casting (c) nonisothermal precision glass molding

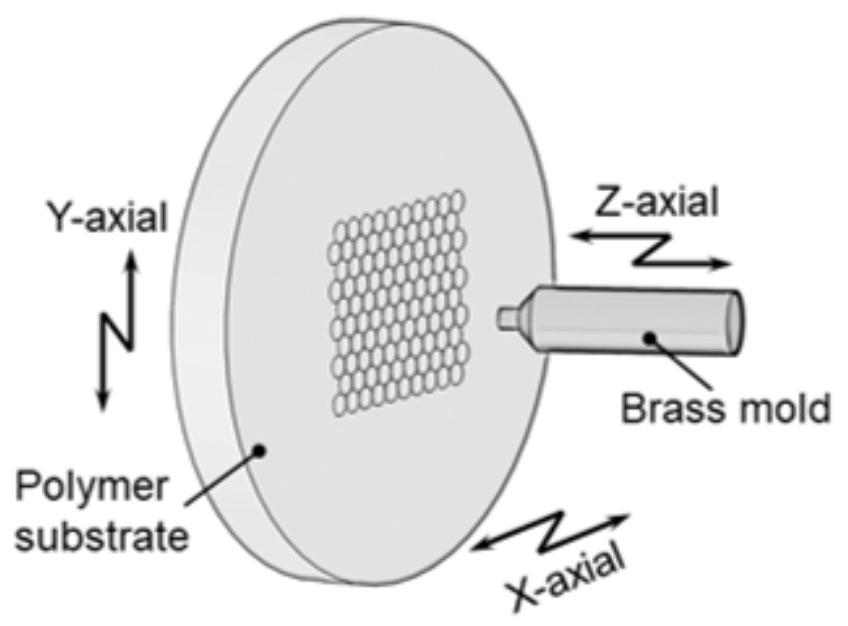

Figure 2

Schematic of the rapid imprinting process
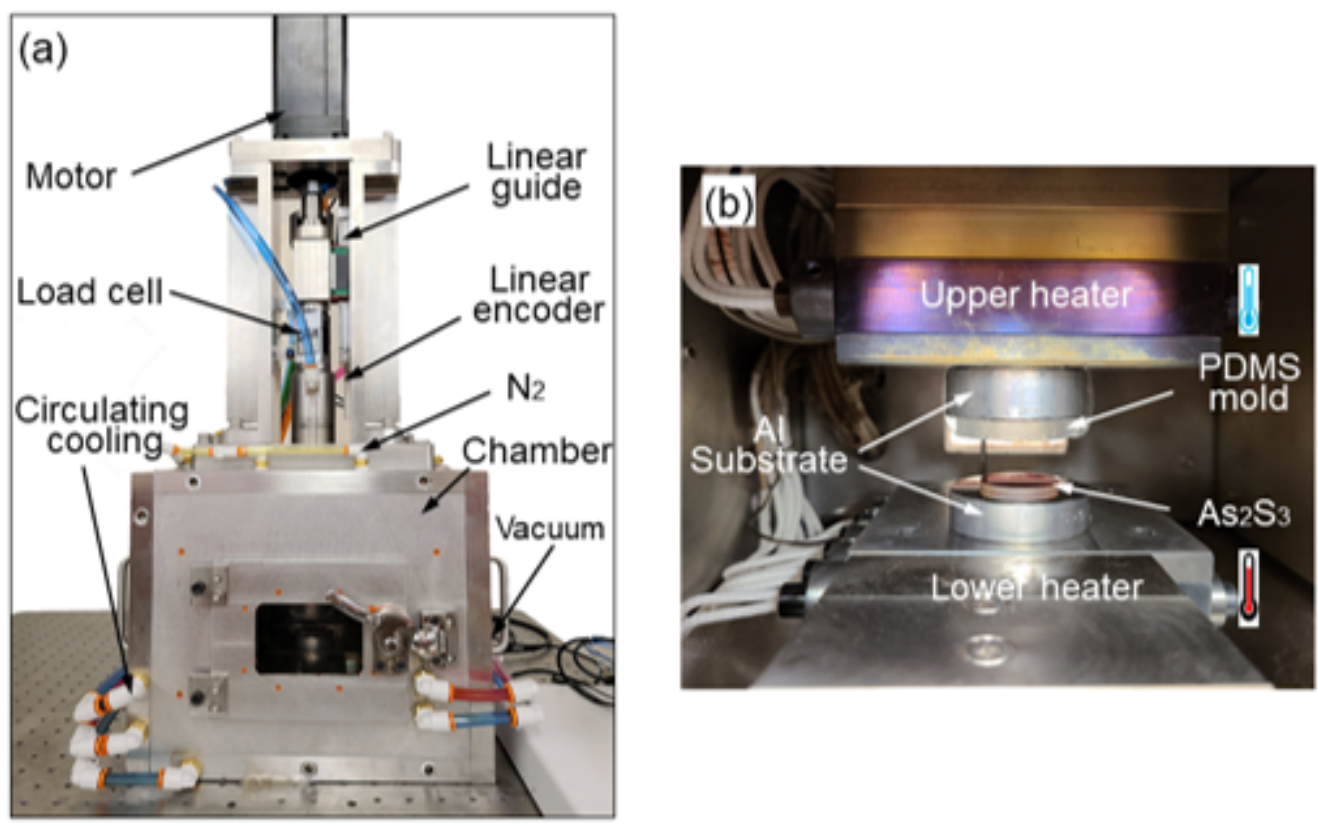

\section{Figure 3}

Photographs of the precision glass molding machine, (a) front view, (b) closeup view of the molding setup 

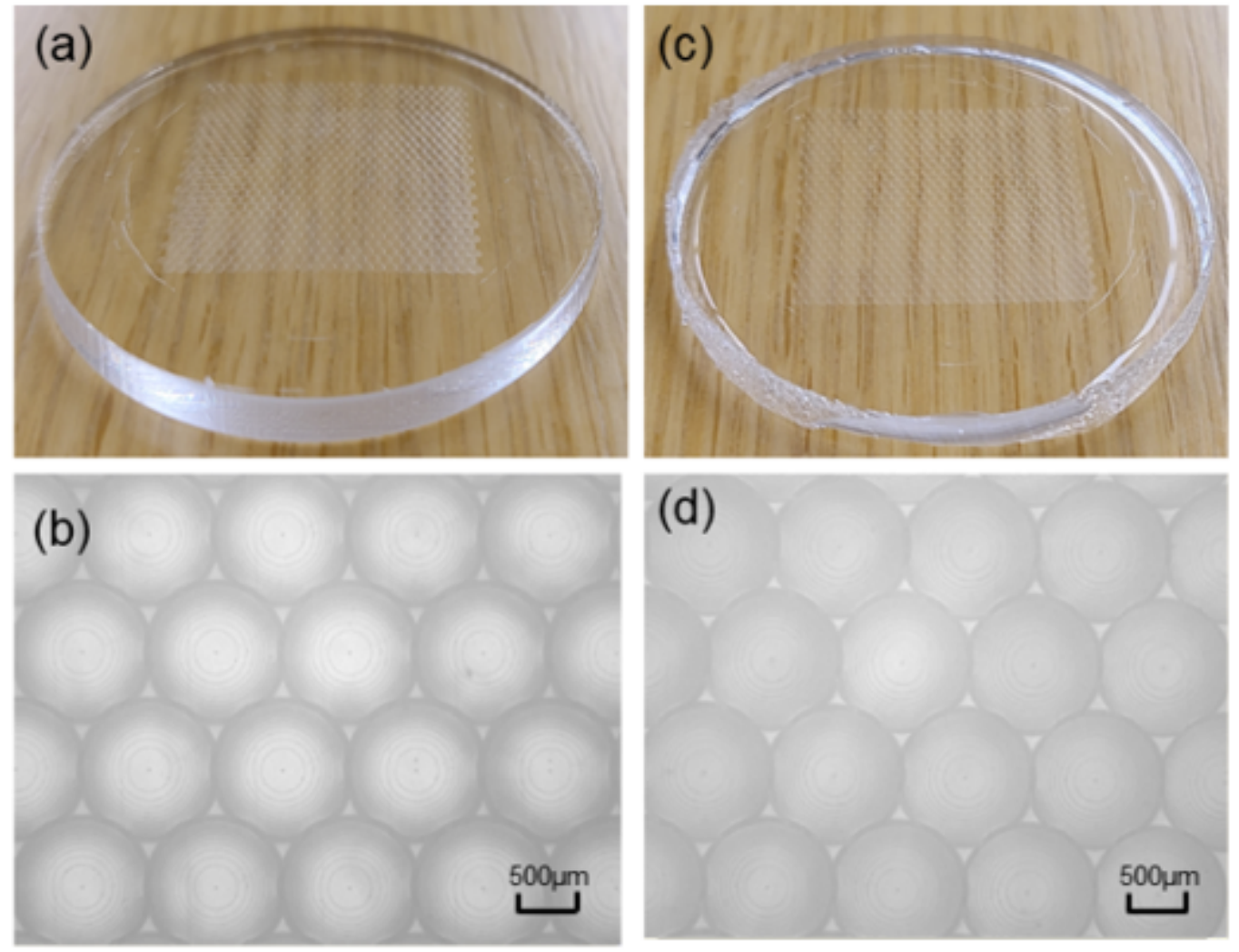

\section{Figure 4}

Photographs of the workpiece and microscope diagrams of the structures on (a) and (b) imprinted PMMA master substrate, (c) and (d) cured PDMS mold 


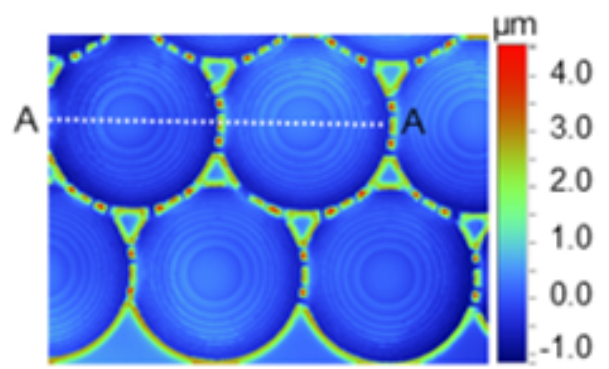

(a)

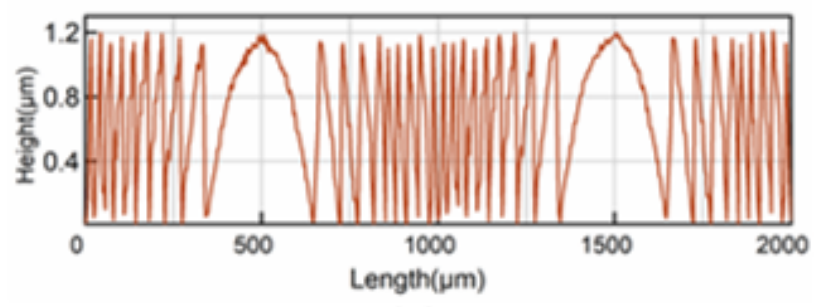

(c)

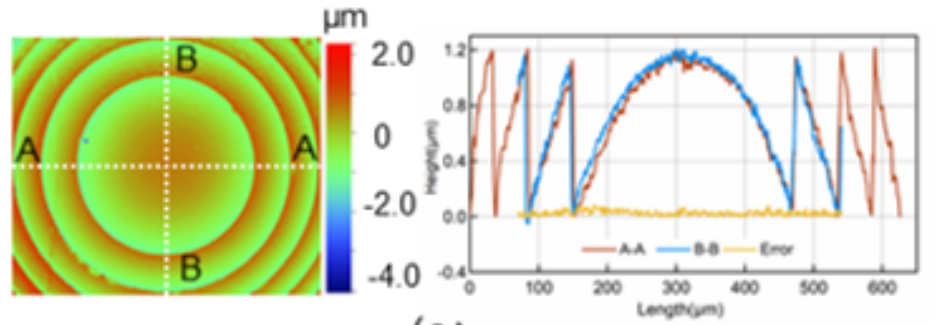

(e)

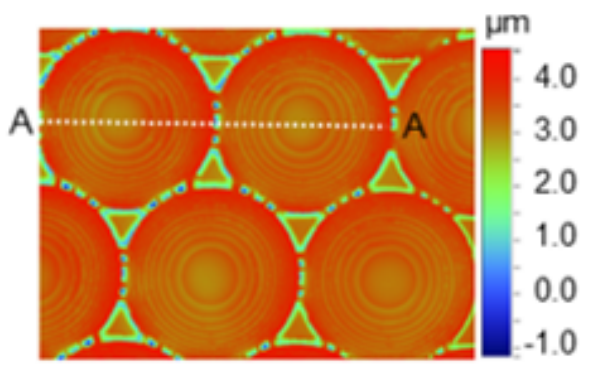

(b)

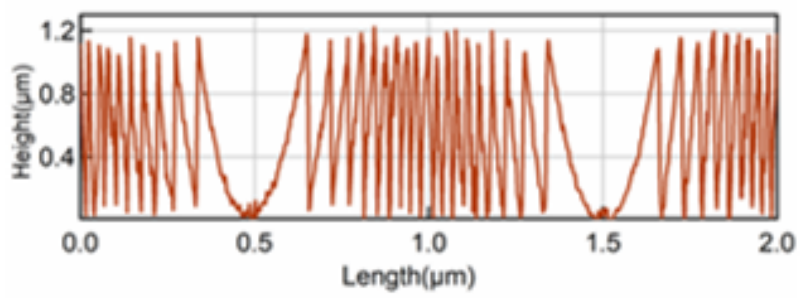

(d)

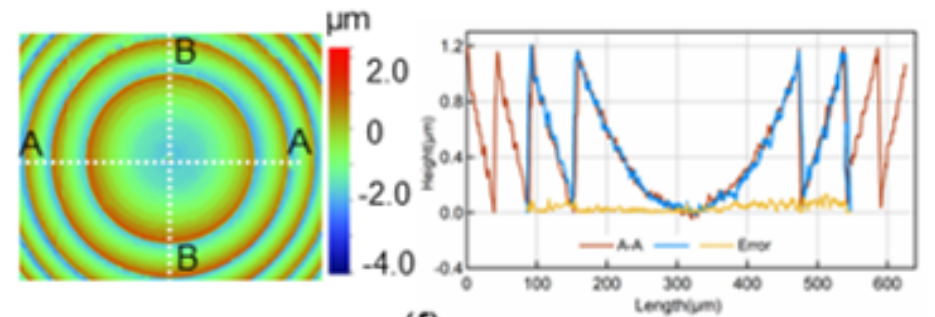

(f)

\section{Figure 5}

The 3D structure of (a) the PMMA master substrate, (b) the PDMS mold, (c), (d) the corresponding profiles along the A-A direction in (a) and (b), the 3D structures and profiles and geometrical errors of the arbitrary lenslet of (e) PMMA master substrate, and (f) PDMS mold 


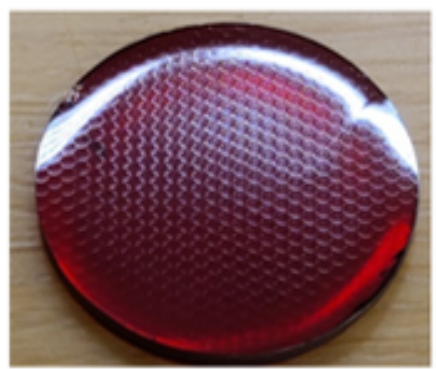

(a)

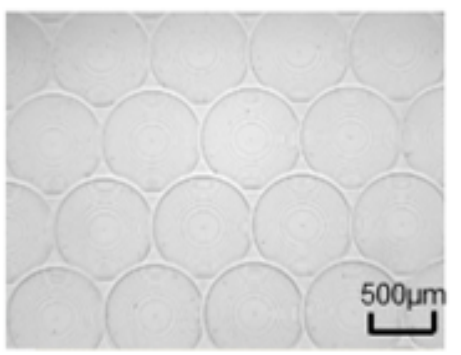

(b)

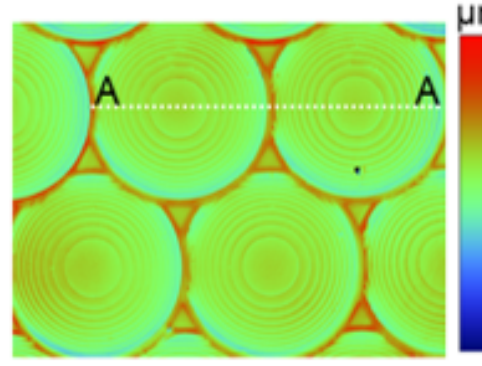

(c)

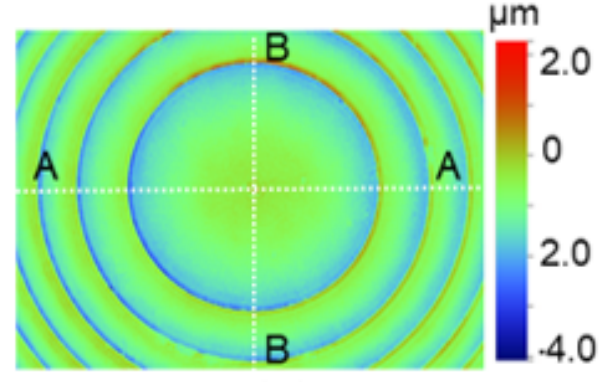

(e)

$\mu \mathrm{m}$
.0

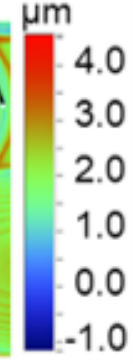

$-1.0$

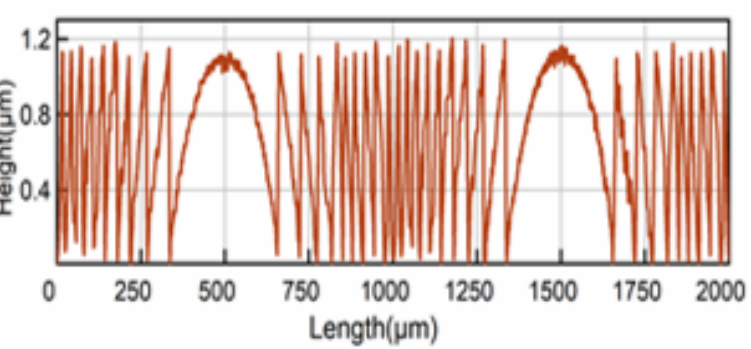

(d)

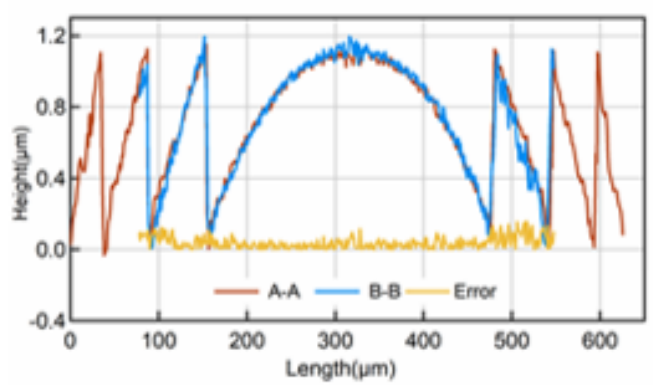

(f)

Figure 6

(a) Photograph and (b) microscope diagrams of the molded diffractive lens array on chalcogenide glass.

(c) the 3D structure of the precision molded diffractive lens array, (d) the corresponding profiles along the A-A direction, (e) 3D structure and (f) profiles and the geometrical errors of an arbitrary lenslet.

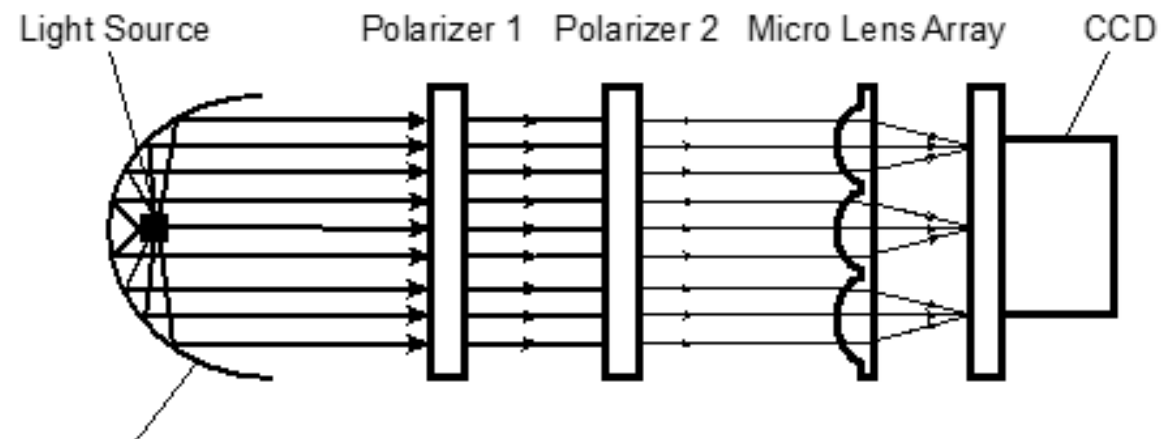

Parabolic Shield

Figure 7

Schematic of optical testing system. 


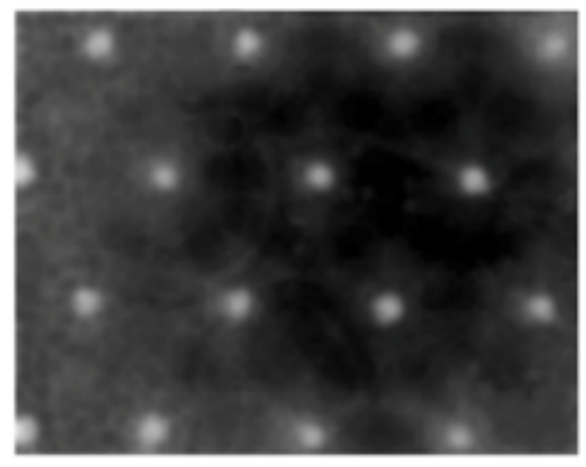

(a)

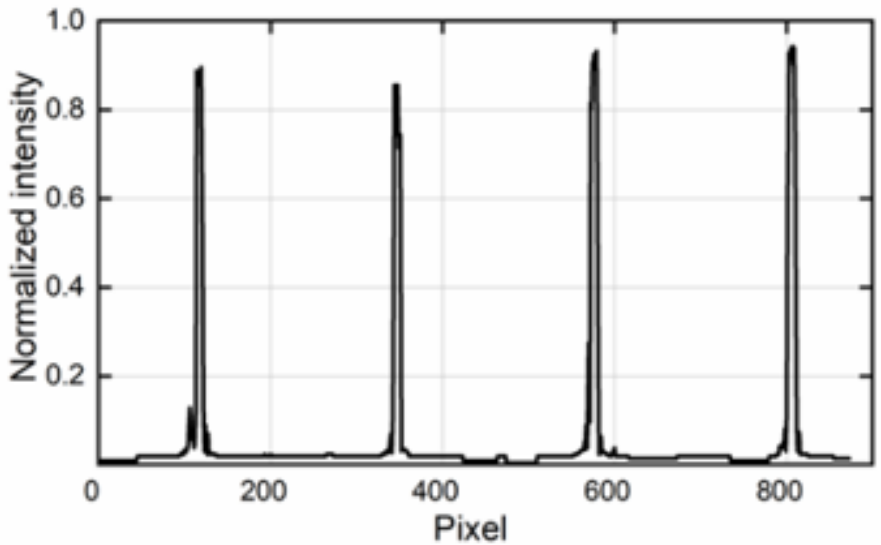

(b)

Figure 8

(a) Focal spots of the $4 \times 4$ Fresnel lens array; (b) focal point intensity distribution of randomly selected a row of microlenslets 